\title{
New Approaches for high lateral resolution Array Tomography analysis
}

Yuuki Yamaguchi ${ }^{1,2}$, Mitsuyo Maeda ${ }^{2}$, Yosky Kataoka ${ }^{2}$, Yukari Moriya ${ }^{1}$,Chikako Nakayama ${ }^{1}$, Tomohiro Haruta ${ }^{1}$, Mitsuo Suga ${ }^{1,2}$, Natasha Erdman ${ }^{3}$ and Shunsuke Asahina ${ }^{1}$

${ }^{1 .}$ JEOL Ltd. Electron Optics Division 1-2 Musashino 3-Chome Akishima TOKYO 196-8558 JAPAN

2. Multi-Modal Microstructure Analysis Unit, RIKEN CLST-JEOL Collaboration Center, Kobe, Japan

3. JEOL USA, INC. 11 Dearborn Road Peabody, MA 01960 USA

Recent applications of Scanning Electron Microscopy (SEM) are versatile. Nowadays, the SEM is not only a surface observation and analysis technique, but can also provide three-dimensional (3D) analysis that collects information about internal structure especially in soft materials such as biological materials. Array Tomography (AT) is one of the methods which are able to analyze large 3D area of biological materials. Ultrathin serial sections prepared with ultra-microtome are placed on substrates and then observed with SEM [1,2]. 3D images are reconstructed by stacking two-dimensional (2D) images of each section. In AT, it is possible to obtain 2D images with high lateral resolution by utilizing an incolumn electron detector. Furthermore, the charging artifacts are substantially reduced, since the substrate on which the sections are placed provides a conductive pathway. This also allows us to perform Energy Dispersive X-ray Spectrometry (EDS) analysis at high incident voltages without charging up. In this report, we present acquisition of 3D images composed of 2D images with high lateral resolution that based on $\mathrm{AT}$ and subsequent acquisition of $3 \mathrm{D}$ elemental maps by AT-EDS method.

Backscattered electron images were obtained with Working Distance (WD) of $2 \mathrm{~mm}$ by using in-column electron detector equipped with energy filter (Upper Electron Detector (UED)) in JEOL FE-SEM. Sample bias was applied in order to improve spatial resolution and detection efficiency of backscattered electrons even at low voltage condition [3]. Backscattered electrons were detected by applying bias on the UED grid as an energy filter. Mouse intercostal muscle tissue was fixed with $2 \%$ glutaraldehyde, $4 \%$ paraformaldehyde and 1\% osmium tetroxide. The resin-embedded tissue was cut at $80 \mathrm{~nm}$ and ultrathin serial sections were placed on silicon wafer or Ultra Flat Carbon (JEOL, Ltd.) substrate that is precisely polished high density amorphous Carbon material. Furthermore, the sections were stained with uranyl acetate and lead citrate and observed with JSM-7800F (JEOL Ltd.) under the following conditions: incident voltage $2.0 \mathrm{kV}$, sample bias $-1.0 \mathrm{kV}$, probe current $3 \mathrm{nA}$, WD $2 \mathrm{~mm}$ or $10 \mathrm{~mm}$, detector UED, UED energy filter bias $1.5 \mathrm{kV}$. The 3D image was reconstructed by stacking 42 serial $2 \mathrm{D}$ images. Elements mapping with high though-put was carried out by using high solid angle multi EDS detectors. Mouse liver tissues were fixed by formaldehyde and immunostained with gold nanoparticles. Then the tissues were stained by uranyl acetate after silver enhancement. The resin-embedded tissues were cut at $100 \mathrm{~nm}$ and serial sections were placed on Ultra Flat Carbon substrate which is one of the best substrate to minimize refraction of backscattered electrons, continuous X-rays and characteristic X-rays from the substrate. Serial sections were analyzed under the following conditions in order to acquire element maps: incident voltage $7.0 \mathrm{kV}$, probe current $1 \mathrm{nA}$, WD $10 \mathrm{~mm}$, multi EDS Detector: two X-max ${ }^{\mathrm{N}} 150$ $\mathrm{mm}^{2}$ and one X-max Extreme $100 \mathrm{~mm}^{2}$ without thin window (Oxford instruments).

Images with WD $2 \mathrm{~mm}$ had much higher lateral resolution than images with WD $10 \mathrm{~mm}$. As the result, it was possible to obtain the 3D image composed of the $2 \mathrm{D}$ images with high lateral resolution. In observation of serial sections, it is necessary to increase scan speed of imaging in order to shorten data 
acquisition time. Large probe current makes it possible to obtain images with high signal to noise ( $\mathrm{S} / \mathrm{N})$ ratio at fast scan speed. However, it results in reduced spatial resolution at large probe current. Therefore, short WD is effective for high lateral resolution AT. In the EDS analysis, an element map of silver nanoparticles in the nucleus was obtained. Although the element map obtained at 10 minutes in live time has high $\mathrm{S} / \mathrm{N}$ ratio, the nanoparticles are visible even in the map obtained at 2 minutes in live time. Element maps are obtained with high throughput by using multi EDS detectors in these experiments. We believe this method is effective for EDS analysis in AT as well.

\section{References:}

[1] K.D.Micheva and S.J Smith, Neuron 55(1) (2007), p.25

[2] Horstmann H et al, PLoS One. 7(4) (2012), e35172.

[3] Suga M et al, Progress in Solid State Chemistry 42 (2014), P.1

(a)

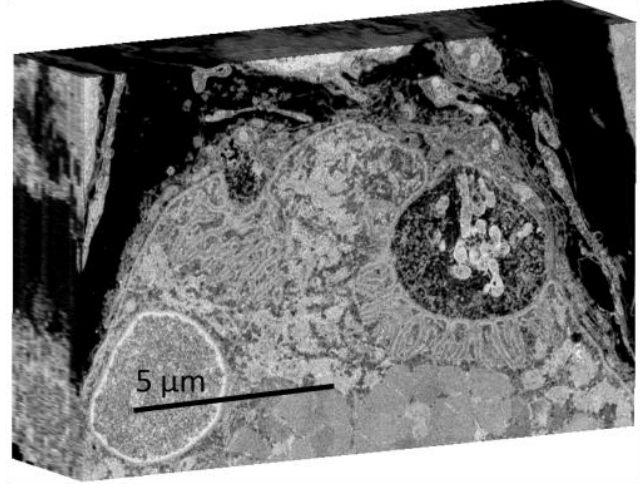

(b)

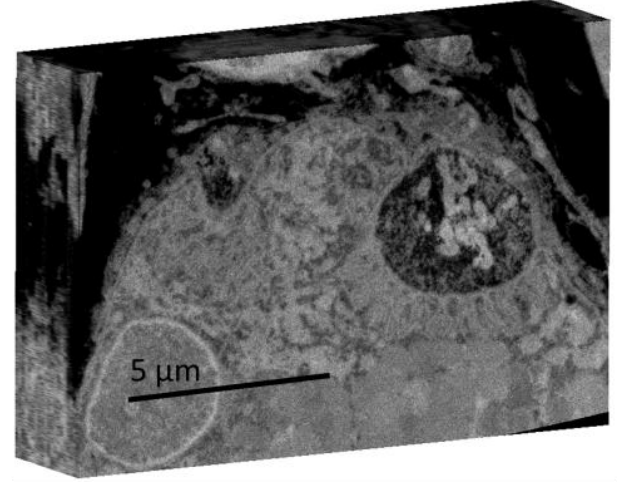

Figure 1. Comparison of images with WD $2 \mathrm{~mm}$ (a) and $10 \mathrm{~mm}$ (b). Scale bar is $5 \mu \mathrm{m}$.

(a)

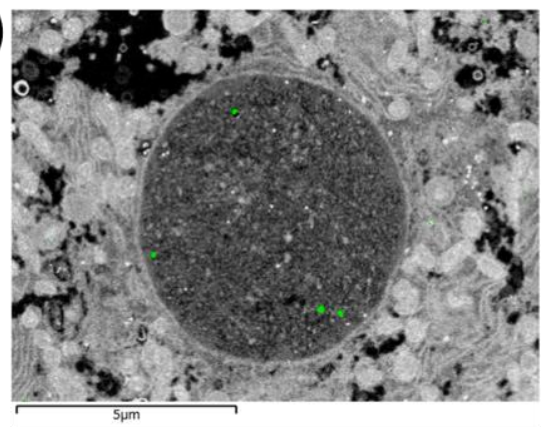

(b)

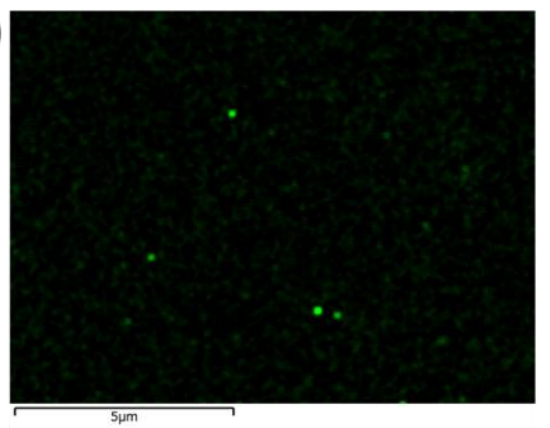

(c)

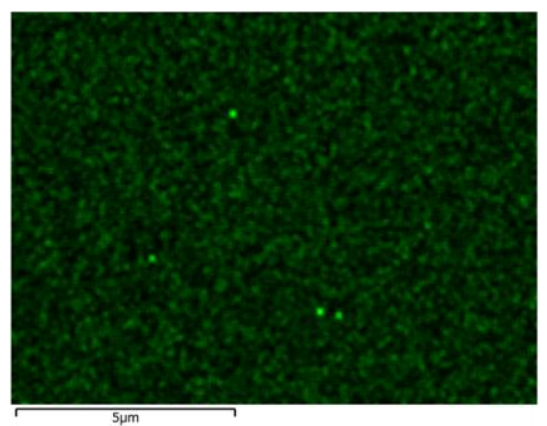

Figure 2. EDS element maps of silver nanoparticles. Backscattered electron image (a) and element maps taken at 10 minutes (b) and 2 minutes (c) in live time. Scale bar is $5 \mu \mathrm{m}$. 